\title{
Derechos Humanos y Género: ejes transversales para fortalecer la gestión universitaria
}

\section{Human Rights and Gender. transversal axes to strengthen university management}

\author{
Verónica Cruz \\ https://orcid.org/0000-0002-5009-2682 \\ veronica.cruz09@yahoo.com.ar \\ Prosecretaría de Derechos Humanos | \\ Universidad Nacional de La Plata | Argentina
}

\section{RESUMEN}

El artículo puntualiza la importancia de transversalizar las perspectivas de derechos humanos y de género en las políticas de gestión universitaria, tomando como referencia la experiencia institucional construida junto al equipo de profesionales que conformamos la Prosecretaría de Derechos Humanos de la Universidad Nacional de La Plata. Inicialmente introduce una reflexión respecto del propio término transversalización, y luego propone algunas ideas desde las cuales andamiar el desafío de trabajar transversalmente los derechos humanos y la cuestión de género en la política universitaria. Por último comparte unas consideraciones de cierre que advierten en torno de la profunda preocupación que suscitan los movimientos negacionistas y la "derechización" de prácticas y discursos que despojan a quienes viven del trabajo, de la posibilidad de ejercer sus derechos en el marco de una convivencia democrática.

\section{ABSTRACT}

The article points out the importance of mainstreaming the perspectives of human rights and gender in university management policies, taking as a reference the institutional experience built together with the team of professionals that make up the Human Rights Office of the National University of La Plata. Initially, it introduces a reflection on the term mainstreaming itself, and then proposes some ideas from which to scaffold the challenge of working across human rights and the gender issue in university politics. Finally, it shares some closing considerations that warn about the deep concern that denial movements arouse and the "right-wing" of practices and discourses that deprive those who make a living from work of the possibility of exercising their rights within the framework of a coexistence democratic.

\footnotetext{
1 Doctora y Magíster en Trabajo Social. Facultad de Trabajo Social, Universidad Nacional de La Plata (FTS UNLP). Especialista en Docencia Universitaria, UNLP. Especialista en Gestión de la Educación Superior. Instituto de Investigación en Educación Superior. Facultad de Odontología, (IIES-FOLP) UNLP. Ex Decana y ex Secretaria Académica de la FTS-UNLP. Profesora Titular Ordinaria de la asignatura "Debate contemporáneo en el Trabajo Social" FTS-UNLP. Actualmente es Prosecretaria de Derechos Humanos UNLP. Investigadora y miembro del Consejo Directivo del Instituto de Estudios en Trabajo Social y Sociedad, FTS-UNLP. Coordinadora Ejecutiva de la Red Interuniversitaria Por la Igualdad de Géneros y Contra las Violencias (RUGE CIN) Docente de posgrado. Compiladora y autora de cuatro libros y de numerosos artículos relacionados con sus temas de investigación.
}

PALABRAS CLAVE

transversalización, derechos humanos, género, Universidad.

\section{KEY WORDS}

mainstreaming, human rights, gender, University. 


\section{INTRODUCCIÓN}

En este artículo reflexionamos acerca de la implicancia de transversalizar las perspectivas de derechos humanos y de género en las políticas de gestión universitaria, desde la experiencia institucional construida junto al equipo de profesionales que conformamos la Prosecretaría de Derechos Humanos de la Universidad Nacional de La Plata.

Experiencia que va materializándose por un lado, al calor de un conjunto de debates y proposiciones que pugnan por redefinir el campo de saberes hegemónicos recuperando voces y producciones de grupos cuyas identidades son invisibilizadas por el imaginario capitalista y patriarcal dominante. Y por otro, en la interlocución con los activismos de los movimientos feministas y de derechos humanos que permean las agendas universitarias con demandas y proyectos en pos de alcanzar una mayor democratización de las instituciones. De este modo, trazamos puentes con nuestro pasado reciente, reconstruyendo memorias que nos permiten reconocer y resignificar los efectos del genocidio como práctica social que transforma estructuralmente la sociedad, construyendo una otredad negativa susceptible de ser hostigada y hasta aniquilada. (Feierstein, 2007)

Si bien los derechos humanos como horizonte de sentido han estado presentes a lo largo de la historia de nuestra Universidad, su abordaje explícito en las políticas institucionales encuentra en las luchas reivindicativas que posibilitaron la reapertura democrática, y en 1986 su normalización y pleno ejercicio de la autonomía y del cogobierno; así como en el amplio debate del año 2008 con la reforma del Estatuto, dos momentos demarcatorios. (Cruz, 2017)

Sin dudas estos acontecimientos han sido y continúan siendo gravitantes para una educación superior comprendida como derecho fundamental que habilita el acceso a otros derechos conquistados en el marco de una dinámica histórica, contradictoria y disputada. De aquí la relevancia de reflexionar en torno de la transversalización como 
dimensión imbricada a procesos de planificación, implementación y evaluación de programas y proyectos de diversa naturaleza. Transversalizar implica necesariamente poner en juego un ejercicio de revisión y problematización de nuestro trabajo en la universidad pública, realizado siempre desde un lugar teórico-epistemológico, político y también geográfico.

\section{Transversalizar implica necesariamente poner en juego un ejercicio de revisión y problematización de nuestro trabajo en la universidad pública, realizado siempre desde un lugar teórico-epistemológico, político y también geográfico.}

Al indagar el alcance del término transversalización, recuperamos los desarrollos realizados en el campo pedagógico principalmente y más recientemente en el campo jurídico-normativo que, si bien son heterogéneos y aluden a dimensiones diferentes, contribuyen a pensar la constitutiva complejidad de la vida social. Es decir, nos brindan un ángulo de lectura para reconocer las múltiples implicaciones e interacciones entre personas, procesos y situaciones sociales, históricas y culturales cuyo abordaje no puede realizarse a través de esquemas perceptivos simplificadores.

En sintonía con la afirmación precedente, transversalizar las perspectivas de derechos humanos y de género y sexualidades no es incorporar algo externo a lo pre-existente, sino producir un cambio radical de lógica, repensar sociopoliticamente escenarios, políticas y prácticas instituyendo umbrales simbólicos de reconocimiento e igualación movilizados por un horizonte emancipatorio. Es también revisar las referencias teóricas y epistemológicas desde las cuales sustentamos el currículum universitario, la investigación y la extensión preguntándonos: ¿qué contenidos enseñamos?; ¿de qué modo lo hacemos?; ¿qué autores, perspectivas y debates ponemos en juego en ese proceso? $; i^{-}$ qué y cómo investigamos? ¿qué lugar damos al cuestionamiento a la desigualdad, al sexismo, a la discriminación, al racismo, a la violencia?; ¿qué herramientas ofrecemos desde el trabajo en las aulas para producir ese ejercicio de reflexividad crítica y llevar adelante prácticas transformadoras?; ¿cómo generamos procesos de doble vía que fortalezcan proyectos que contribuyan a mejorar la vida de los sectores que viven del trabajo? Estas preguntas no pueden ser abordadas con respuestas unívocas ni definitivas, por el contrario, las enunciamos para mantener lo preguntado en su problematicidad, sin clausurar la disputa por el sentido que nos moviliza a tomar decisiones e intervenir recorriendo el desafío de habitar el presente desde las interpelaciones del pasado. 
¿qué contenidos enseñamos?; ¿de qué modo lo hacemos?; ¿qué autores, perspectivas y debates ponemos en juego en ese proceso? ¡¿qué y cómo investigamos? ¿qué lugar damos al cuestionamiento a la desigualdad, al sexismo, a la discriminación, al racismo, a la violencia?; ¿qué herramientas ofrecemos desde el trabajo en las aulas para producir ese ejercicio de reflexividad crítica y llevar adelante prácticas transformadoras?; ¿ cómo generamos procesos de doble vía que fortalezcan proyectos que contribuyan a mejorar la vida de los sectores que viven del trabajo? Estas preguntas no pueden

En un contexto de "derechización," negacionismo y pandemia que, tal como expresa Jorge Aleman (2021), propone un presente absoluto que destruye "los puntos de anclaje, los puntos de amarre de la significación que hacen posible la lectura de los legados históricos y las herencias simbólicas," recorrer estos interrogantes se vuelve un imperativo ético y político para la universidad pública. $O$ dicho en otros términos, cuando la apuesta es formar profesionales comprometidos con la construcción de universidades y sociedades más justas e igualitarias, las narrativas que evocan una memoria crítica, compleja, mediatizada por una pedagogía que propicie el des-ocultamiento de verdades históricas y vehiculice su constitución como sujetos políticos, deviene una operatoria fundamental.

En virtud de estas consideraciones, entendemos que la preocupación por la transversalización de las perspectivas de género y de derechos humanos surge y se profundiza al identificar la producción de ciertas ausencias que devienen problemáticas a la hora de fortalecer un proyecto de universidad pública, popular, democrática e inclusiva. Desnaturalizar esas ausencias exige "hacer las cuentas" con nuestras tradiciones, interrogar instituidos, visibilizar lo invisibilizado, y generar propuestas situadas, que reafirmen la potencialidad y exigencia de este trabajo de elucidación. (Castoriadis, 2007)

Cerramos estas notas introductorias reafirmando el papel de la educación superior en la conformación y en el reconocimiento de las identidades, que exige desnaturalizar las formas dominantes, androcéntricas y patriarcales en las que circula y se ejerce el poder que produce su silenciamiento e invisibilización. Y compartimos en los apartados siguientes, algunas puntualizaciones respecto del tema al que nos invita esta edición de la revista Trayectorias; particularizando las reflexiones en torno de las perspectivas mencionadas desde las experiencias en curso en nuestra universidad, sin desconocer la indispensable interlocución de los enfoques de derechos humanos, de igualdad de género, de discapacidad, interculturalidad e intergeneracional. 


\section{HACIA LA TRANSVERSALIZACIÓN DE LA PERSPECTIVA DE DERECHOS HUMANOS}

Pensar la universidad pública y las prácticas de quienes trabajamos y estudiamos en ella en clave de derechos humanos, exige por un lado, una referencia crítica y situada acerca del pasado reciente signado por "las grandes tragedias del siglo XX que muestran cómo aquellas ideas modernas de un sujeto racional, omnipotente y de progreso, son puestas en jaque frente a la violencia y el horror patriarcal-colonial que produce la experiencia práctica, ética y política de los fascismos, el estalinismo, la guerra, los campos de concentración y exterminio." (Cruz y Morandi, 2021, p. 17) Y por otro, un trabajo colectivo que movilice y sostenga un ejercicio de memoria para comprender el horror y el carácter extremo de las violencias perpetradas por los genocidas sobre los pueblos, capturando sus puntos de fisura para desde allí, enseñar, aprender y reflexionar acerca de la relación paradójica y singular que establecemos con nuestro tiempo.

Sólo de ese modo podremos fundar -desde el acto educativo- núcleos de sentidos contrahegemónicos, reconociendo los efectos del genocidio en nuestro país y la región, para que no se repita. Y aquí la educación superior tiene una responsabilidad y una potencialidad social, cultural y política insoslayable, convocándonos a reconocer la dialéctica pasado-presente-futuro como proceso de emancipación y autonomía viabilizado por la transmisión inter-generacional de una herencia.

En esta dirección, es posible afirmar que el tratamiento de los derechos humanos en la universidad se da estrechamente ligado a procesos históricos de luchas protagonizadas por actores sociales comprometidos con la formación política e intelectual. Asimismo, esa inscripción da lugar a su progresiva institucionalización como campo que atraviesa las prácticas universitarias, interpelando los discursos dominantes y las realidades de desigualdad estructural en la que viven millones de personas. Institucionalización que si bien remite a tradiciones y regularidades que dan sentido y significación a experiencias singulares; también posibilita -en un movimiento tensionado- la emergencia de lo nuevo y refuerza el horizonte formativo emancipatorio que aspiramos construir. 
Coincidimos con Abrate cuando afirma que "la problemática de los derechos humanos constituye en sí misma un eje transversal de la formación, aunque ha sido siempre difícil su inclusión en instancias formales. Las características de los planes de estudio y el énfasis disciplinar y profesional de las carreras universitarias suele obturar el desarrollo de estas temáticas." (2019, p.68) De aquí la relevancia de desplegar un pensar situado, que dé cuenta del modo en que nuestras subjetividades contemporáneas son configuradas por el pasado reciente, transversalizando esta perspectiva como sustrato de la formación y la intervención socio-profesional, para comprender los problemas sociales que la dominación neoliberal fabrica sin tregua. Esa praxis, retroalimentada por la producción de conocimientos, pone en cuestión los mecanismos de negación y olvido, la desigualdad y el avasallamiento de los derechos humanos, dimensiones ineludibles para interpelar y fortalecer lo público.

En sintonía con este planteamiento, afianzar la perspectiva de derechos humanos como principio rector de la vida universitaria, conlleva a valorar el carácter sustancial que, en todo acto pedagógico, reviste la lucha por el sentido. Es precisamente el trabajo reflexivo y propositivo en torno de los fundamentos éticos, teóricos y políticos, identificando las matrices y memorias que moldean las significaciones imaginarias sociales (Castoriadis, 2007) y animan nuestro quehacer, el que posibilita constituirnos como comunidad de iguales, respetando nuestras diferencias y haciendo emerger tradiciones enmudecidas.

\section{afianzar la perspectiva de derechos humanos como princi- pio rector de la vida universitaria, conlleva a valorar el ca- rácter sustancial que, en todo acto pedagógico, reviste la lucha por el sentido.}

Para finalizar este apartado, mencionamos de manera abreviada que este trabajo en nuestra universidad viene siendo desarrollado desde la Dirección de Políticas de Memoria y Reparación dependiente de la Prosecretaría de Derechos Humanos, junto a las áreas institucionales de derechos humanos de cada Unidad Académica. Trabajamos con diferentes estrategias que pugnan por resignificar los efectos del genocidio perpetrado por las fuerzas militares y de seguridad sobre nuestra institución. Ese recorrido es construido en una doble dimensión pedagógica: en la reparación documental, focalizada en legajos y registros de información sobre las casi 800 personas detenidas desaparecidas o asesinadas desde momentos previos a la última dictadura; y en la progresiva curricularización de los derechos humanos en la formación de grado y pregrado, además de promover desarrollos investigativos y extensionistas².

2 Para ampliar ver Godoy, G. (2020) Sistematización de la experiencia del programa "Reparación, digitalización y preservación de legajos de estudiantes, graduadxs y trabajadorxs de la UNLP víctimas del Terrorismo de Estado" TFI presentado en el marco de la Especialización en Gestión de la Educación Superior IIES-FOLP UNLP. en http://sedici.unlp.edu.ar/handle/10915/115279 
En el sistema universitario público, y en particular en nuestra Universidad, en los últimos años vienen generándose condiciones y proyectos sumamente valiosos en materia de igualdad y de erradicación de las violencias de género, con la instalación de debates acerca de las formas de la discriminación y violencia que diera lugar a la creación de Protocolos de Actuación. (Torlucci, Vazquez Laba y Pérez Tort, 2019) Estos cambios se gestan en un escenario de fuerte protagonismo de los feminismos donde la conformación del Movimiento Ni una Menos en junio de 2015, los pañuelazos y las luchas por la legalización del aborto, operan como acontecimientos demarcatorios.

Este contexto interpela a la comunidad universitaria, visibilizando las violencias sexistas que se reproducen en nuestras instituciones académicas y planteando la necesidad de crear desde la gestión, planes y políticas de igualdad, y dispositivos que contrarresten la desigualdad; impulsando la transversalización de género en las prácticas de formación, de extensión y de investigación.

Cabe aclarar que este debate y las propuestas que surgen a partir del mismo, parte de considerar que habitamos entramados institucionales producidos e inscriptos en la organización social, económica, política y cultural que reconocemos como sistema de dominación patriarcal (Millet, 1969). Este sistema subordina el sexo femenino al masculino, vulnerando los derechos de las mujeres y disidencias sexo-genéricas y naturalizando esas asimetrías. Siendo esto así, la creación de políticas y programas institucionales que visibilicen los efectos del mismo, aborden las resistencias y remuevan obstáculos se torna un objetivo a alcanzar en el corto plazo.

Comprendemos que la organización social capitalista patriarcal es movilizada por estructuras de poder y dominio que reproducen y legitiman la expropiación de los cuerpos, bienes y principalmente de la capacidad de decidir de las mujeres, realizada por los hombres. (Federici, 2010) Frente a esta realidad es necesario advertir que las relaciones antagónicas de clase y de género que devienen del movimiento de dominación sobre los cuerpos de las mujeres e identidades feminizadas, resultan estratégicas para asegurar la acumulación capitalista.

Dicha lógica sitúa a las mujeres en una posición de desventaja ocultando deliberadamente esa operatoria social histórica, y jerarquiza las diferencias que atraviesan y moldean dinámicas institucionales y campos de conocimiento, tal como lo demostraran los desarrollos epistemológicos críticos realizados hace décadas por feministas de diferentes vertientes, visibilizando el carácter sexista, androcéntrico y colonial de la ciencia moderna.

De aquí nuestra insistencia en desplegar un trabajo de problematización de los efectos que las desigualdades producen en quienes habitamos la institución universitaria, impregnando todas las actividades que desarrollamos. Una praxis feminista que lea e intervenga ante la reconfiguración del poder, reafirmando el papel estratégico de la gestión de políticas de igualdad que propicien la transversalización de la pers- 
pectiva de genero desde una mirada interseccional.

Es decir, políticas que atraviesen y reformulen el currículum y la producción de conocimientos para reorientar desde esta perspectiva, la formación profesional en todos los campos disciplinares, poniendo en tensión las construcciones androcéntricas y heteronormativas que reproducen desigualdades de clase, de género, generacionales, raciales, entre otras.

Asumirnos en esta tarea implica perforar el entramado de relaciones de nuestras instituciones universitarias, hacerlas más permeables a las articulaciones con el movimiento feminista y de las disidencias para incorporar sus saberes y demandas.

\section{Asumirnos en esta tarea implica perforar el entramado de relaciones de nuestras instituciones universitarias, hacer- las más permeables a las articulaciones con el movimiento feminista y de las disidencias para incorporar sus saberes y demandas.}

Bajo esta premisa, podemos mencionar el trabajo que se desarrolla desde las Direcciones de Género y Diversidad y de Políticas Feministas dependiente de la Prosecretaría de Derechos Humanos de esta universidad, mediante acciones vinculadas a: la capacitación y formación permanente en género, enmarcada en la implementación de la Ley Micaela; la creación de unidades de atención que receptan consultas y/o denuncias por situaciones de violencia de género, coordinados por equipos profesionales que brindan acompañamiento a las personas afectadas por las mismas; la implementación de acciones de inclusión que favorezcan las trayectorias educativas y laborales de personas con identidades sexo-genéricas disidentes; la construcción de estrategias tendientes a reflexionar sobre las masculinidades; el trabajo en el marco de la implementación de la Ley de Educación Sexual Integral y de las recientes leyes de Interrupción Legal del embarazo y de inclusión laboral de personas trans, entre otras. A estas propuestas sumamos también por un lado, acciones tendientes a generar mayor paridad en los cargos de gestión y de docencia e investigación universitaria; así como en la selección y lectura de bibliografía que sustenta los programas de las asignaturas y los planes de estudios. Y por otro, la progresiva incorporación de políticas de cuidado que permitan construir trayectorias educativas y laborales más inclusivas e igualitarias.

Sin dudas estas iniciativas -entre otras- fortalecen el entramado relacional de nuestras comunidades universitarias, poniendo en juego el componente de politización inherente al campo de los derechos humanos y al género en particular. No obstante, cuando la apuesta es transversalizar la perspectiva de género de modo tal que la universidad pueda reconocerse y afianzarse como un territorio plural y democrático, la reconstrucción de la memoria y la crítica a los saberes hegemónicos desde una pedagogia feminista es una operatoria estra- 
tégica, en tanto redefine los principios de justicia y posibilita construcciones para una educación no sexista ni racista.

Los movimientos pedagógicos y los feminismos vuelven la mirada sobre los procesos y formas de opresión y dominación porque participan en la reconstrucción de la memoria. Esta relación entre feminismos y memoria permite la producción de conocimiento situado toda vez que rescata del olvido las experiencias de las mujeres y demás grupos oprimidos, pero también sus historias y sus voces. Un trabajo de memoria que busca recuperar las experiencias pedagógicas emancipadoras en defensa de les oprimides, silenciades y discriminades por el patriarcado, y reconocer la participación de movimientos y organizaciones de trabajadores y estudiantes en una historia de lucha y disputa por el sentido de la educación pública, y en defensa de la educación como un derecho y del derecho a la educación. (Seoane, 2021, p. 44).

Sintetizando, podemos afirmar que la transversalización del género en la vida universitaria promueve la igualdad sólo si se imbrica sistemáticamente a las estructuras, las políticas, los procesos y procedimientos que intervienen en la organización institucional. Ese atravesamiento genera cambios sustantivos e indispensables en los estándares asociados a la masculinidad y feminidad, y en su valoración -producto de las inercias de género sedimentadas en el aparato estatal y perpetuadas mediante sus regularizaciones e intervenciones.(Rodríguez Gustá, 2008, p. 115) De aquí la importancia de reconocer la historicidad imbricada a la edificación simbólica y material de nuestras instituciones, donde las jerarquías de género son demarcatorias y su revisiòn crítica no puede realizarse de modo simple ni en el corto plazo, sino mediante procesos de transversalización que a mediano y largo plazo permitan contar con una currícula feminista, con presupuestos universitarios definidos desde una economía feminista, con investigaciones fundamentadas en una epistemología feminista, con infraestructura universitaria con perspectiva de género e inclusiva.

\section{CONSIDERACIONES FINALES}

Las reflexiones compartidas en este artículo son escritas en un contexto de capitalismo pandémico (Butler, 2020), en el que vemos con profunda preocupación el modo en que las derechas hacen causa común en una serie de estrategias y dispositivos para enfrentar la "inseguridad," instalando un discurso punitivo y crecientemente xenófobo al referirse principalmente a sectores excluidos del empleo formal, y a mujeres e identidades sexo-genéricas disidentes. Esta cosmovisión neoliberal conservadora legítima y amplía los márgenes de acción de las fuerzas de seguridad sobre personas empobrecidas, inmigrantes, pueblos originarios; mujeres; infancias vulnerabilizadas entre otros grupos, despojándolos de la posibilidad de ejercer sus derechos y de vivir una vida digna.

Frente a esta realidad procuramos contribuir con la ampliación de marcos de reconocimiento; con la institucionalización transversal de 
los derechos humanos en la cultura universitaria, desde una mirada interseccional que dé cuenta e intervenga ante las múltiples opresiones. Un trabajo político institucional que identifique los puntos de tensión en la educación superior, a fin de producir transformaciones. Para decirlo de manera más precisa, es fundamental poner al descubierto las formas que reviste la dominación patriarcal-capitalista-colonial desde un ejercicio de reflexividad crítica consistente que, al asumir el horizonte de expectativas no cumplidas del pasado, posibilite forjar proyectos e itinerarios colectivos e individuales más justos e igualitarios.

\section{Frente a esta realidad procuramos contribuir con la amplia- ción de marcos de reconocimiento; con la institucionaliza- ción transversal de los derechos humanos en la cultura uni- versitaria, desde una mirada interseccional que dé cuenta e intervenga ante las múltiples opresiones. Un trabajo políti- co institucional que identifique los puntos de tensión en la educación superior, a fin de producir transformaciones.}

Para finalizar, entendemos que la apuesta es producir desde la universidad pública, un movimiento instituyente, contrahegemónico, desde un pensamiento y una práctica que ilumine las divergencias e interrogue las sedimentaciones de significados instituidos, generando condiciones que efectivicen la educación como derecho. Pero ese movimiento sólo es posible si potenciamos la capacidad de conmover-nos, indignarnos e interpelar-nos ante la desigualdad y la consecuente precarización de la existencia humana. Es decir, si repolitizamos el acto educativo recreando el carácter público, autónomo y co-gobernado de nuestra universidad, desde un diálogo intercultural, plural y democrático, sin reminiscencias colonizadoras ni moralizantes. 


\section{BIBLIOGRAFIA}

Abrate, J. P. (2029). Derechos Humanos y Educación Superior. Capítulo en Badano, M. R (comp). Educación Superior y Derechos Humanos. Reflexiones, apuestas y desafíos. Editorial UADER, CIN_RIDDHH. Paraná, Entre Ríos, Argentina. (pp 67-77)

Aleman, J. (2021). Es un deber ético que no haya negacionismo, ni genocidio, ni el delito de lesa humanidad o la explotación que lo hacen posible. (en línea) https://www.hijos-capital.org.ar/2021/03/28/es-un-deber-etico-que-no-haya-negacionismo-ni-genocidio-ni-el-delito-de-lesa-humanidad-o-la-explotacion-que-lo-hacen-posible/

Butler, J. (2020). El capitalismo pandémico hace desechable la vida de los trabajadores. En Milenio https://www.bing.com/search?q=capitalismo\%20pandemico\%20butler\&pc=0MOX\&ptag=C10N1000AD053135386\&form=CONBDF\&conlogo=CT3210127\#: :text=el\%20capitalismo\%20pand\%C3\%A9mico-,https\%3A// www.milenio.com/cultura/judith-butler-el-coronavirus-y-el...,-30/5/2020

Castoriadis, C. (1997-2007). La institución imaginaria de la sociedad 1a. Ed. Buenos Aires: Tusquets Editores.

Cruz, V y Morandi, G. (2021). Educación superior, derechos humanos y género: claves para repensar la Universidad pública. Cap. 1 en Cruz, López y Luquet (coord.) Transversalizar la perspectiva de género. Aportes desde una experiencia colectiva en el ingreso universitario 1a ed. - La Plata : EDULP, 2021. Libro digital, PDF.

Cruz, V. (2017). La universidad de los debates en https://www.debates.unlp. edu.ar/2017/03/09/universidad-y-derechos-humanos/

Feierstein, D. (2007). El genocidio como práctica social. Fondo de cultura económica. Buenos Aires.

Godoy, G. (2020). Sistematización de la experiencia del programa "Reparación, digitalización y preservación de legajos de estudiantes, graduadxs y trabajadorxs de la UNLP víctimas del Terrorismo de Estado" TFI presentado en el marco de la Especialización en Gestión de la Educación Superior IIES-FOLP UNLP. en http://sedici.unlp.edu.ar/handle/10915/115279

Millet, K. (1995). Política Sexual. Madrid: Cátedra.

Rodríguez Gustá, A. (2008). Las políticas sensibles al género: variables conceptuales y desafíos de intervención. En Temas y debates, (16), 109-129

Torlucci, S.; Vazquez Laba, V.; Pérez Tort, M. (2019). La segunda reforma Uni- 
versitaria: políticas de género y transversalización en las universidades. Revista Científica de la Red de Carreras de Comunicación Social (REVCOM). https://perio.unlp.edu. ar/ojs/index.php/revcom/article/view/5742 